\title{
Hadron-quark continuity induced by the axial anomaly in dense QCD
}

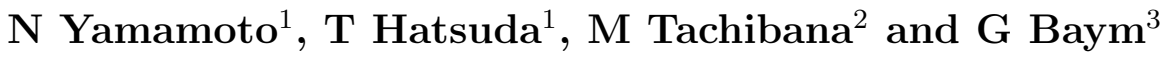 \\ ${ }^{1}$ Department of Physics, University of Tokyo, Japan \\ ${ }^{2}$ Department of Physics, Saga University, Saga 840-8502, Japan \\ ${ }^{3}$ Department of Physics, University of Illinois, 1110 W. Green St., Urbana, Illinois \\ 61801, USA \\ E-mail: yamamoto@nt.phys.s.u-tokyo.ac.jp
}

\begin{abstract}
.
We investigate the interplay between the chiral and diquark condensates on the basis of the Ginzburg-Landau potential with QCD symmetry. We demonstrate that the axial anomaly drives a new critical point at low temperature in the QCD phase diagram and leads to a smooth crossover between the hadronic and color superconducting phases.
\end{abstract}

\section{Introduction}

Unravelling the phase structure in the intermediate baryon density region between the hadronic and deconfined phases is one of the key issues in quantum chromodynamics (QCD). The phase structure is also relevant to the matter in the interiors of neutron and possible quark stars and to the dynamics of moderate energy heavy-ion collisions in the future at GSI.

Recently, we have shown the existence of a new critical point and an associated smooth crossover at intermediate density region in a model-independent GinzburgLandau (GL) analysis of the phase structure [1, 2]. The smooth crossover is intimately connected to the idea of hadron-quark continuity, proposed in 3. In this analysis, the coupling between the chiral condensate $\Phi \sim\langle\bar{q} q\rangle$ and the diquark condensate $d \sim\langle q q\rangle$ plays a crucial role.

\section{Ginzburg-Landau potential}

The guiding principle in constructing the GL potential describing chiral and diquark condensates is the preservation of the QCD symmetry, $\mathcal{G} \equiv S U(3)_{L} \times S U(3)_{R} \times U(1)_{B} \times$ $U(1)_{A} \times S U(3)_{C}$, where $U(1)_{A}$ is explicitly broken down to $Z_{6}$ by the axial anomaly. We define the chiral condensate $(\Phi)$ and diquark condensate $(d)$ in the $J^{P}=0^{+}$channel by $\Phi_{i j} \sim-\left\langle\bar{q}_{R}^{j} q_{L}^{i}\right\rangle$ and $\left\langle\left(q_{L}\right)_{b}^{j} C\left(q_{L}\right)_{c}^{k}\right\rangle \sim \epsilon_{a b c} \epsilon_{i j k}\left[d_{L}^{\dagger}\right]_{a i}$ (plus $L \leftrightarrow R$ ), where $i, j, k$ and $a, b, c$ 
are the flavor and color indices, and $C=i \gamma^{2} \gamma^{0}$ is the charge conjugation operator. The order parameters transform under $\mathcal{G}$ as $\Phi \rightarrow \mathrm{e}^{-2 i \alpha_{A}} V_{L} \Phi V_{R}^{\dagger}, d_{L} \rightarrow e^{2 i \alpha_{B}+2 i \alpha_{A}} V_{L} d_{L} V_{C}^{\mathrm{t}}$, where the $V_{L, R, C}$ are $S U(3)_{L, R, C}$ rotations and the $\alpha_{A, B}$ are $U(1)_{A, B}$ rotations. Given these tranformations, and assuming that the order parameters are small enough to write a power series, we can construct the most general GL potential $\Omega(\Phi, d)$ invariant under $\mathcal{G}$ except for $U(1)_{A}$. For three massless flavors, after making an ansatz of maximal flavor symmetry $\left(\Phi=\operatorname{diag}(\sigma, \sigma, \sigma)\right.$ and $\left.d_{L}=-d_{R}=\operatorname{diag}(d, d, d)\right)$, the general GL potential assumes the simple form up to fourth order in $\Phi$ and $d$,

$$
\Omega_{3 \mathrm{~F}}=\left(\frac{a}{2} \sigma^{2}-\frac{c}{3} \sigma^{3}+\frac{b}{4} \sigma^{4}+\frac{f}{6} \sigma^{6}\right)+\left(\frac{\alpha}{2} d^{2}+\frac{\beta}{4} d^{4}\right)-\gamma d^{2} \sigma+\lambda d^{2} \sigma^{2},
$$

where all the coefficient terms depend on temperature, $T$, and chemical potential, $\mu$. Since $|\beta| \gg|\lambda|$, as can be shown from microscopic theories such as weak coupling QCD and the Nambu-Jona-Lasinio (NJL) model, we treat the $\lambda$-term as a small perturbation in the three-flavor case. On the other hand, $\gamma$ and $c$, which both originate from the axial anomaly, are not negligible. Indeed, a positive $c$ is essential for making the $\eta^{\prime}$ meson much heavier than the pion; it also leads to a first-order chiral phase transition at finite $T$ with $\mu=0$. In equation (1), the $\gamma$-term acts as an external field for $\sigma$ and leads to a new critical point as we see below.

In principle, the system can have four possible phases: normal (NOR) $(\sigma=d=0)$, $\operatorname{CSC}(\sigma=0, d \neq 0)$, NG $(\sigma \neq 0, d=0)$, and coexistence (COE) $(\sigma \neq 0, d \neq 0)$. We locate the phase boundaries and the order of the phase transitions within the GL formalism by comparing the potential minima in these phases.

\section{Phase structure}

Figure 1 shows the phase structure in the $a$ - $\alpha$ plane for the massless three-flavor case with and without the $\gamma$-coupling $\ddagger$ The figure shows the case for $b>0$; the structure for $b<0$ is not qualitatively different. Without the $\gamma$-coupling, the critical lines of the chiral and the CSC transitions simply cross (left panel of Fig. 1). With the $\gamma$-coupling, the phase structure undergoes major modifications. Firstly, the first order line between the CSC and COE phases, which originally continued all the way down terminates at a critical point; as a result, the CSC and COE phases are continuously connected. This is because the $\gamma$-term acts as an external field for $\sigma$, smoothing out the first order chiral transition for large $\gamma d^{2}$. Moreover, the second order CSC transition splits into two, since $\sigma$ varies discontinuously across the first order line between the NOR and COE phases.

Mapping the coordinates from $(a, \alpha)$ to $(T, \mu)$, we obtain the schematic phase diagram for massless three-flavor shown in the left panel of Fig. 2. Analyzing the

massless two-flavor case similarly, we arrive the phase diagram in the right panel of Fig. 2.

$\ddagger$ For $\gamma>(c / 3) \sqrt{\beta / b}$, a tricritical point emerges on the boundary between the NG and COE phases. 

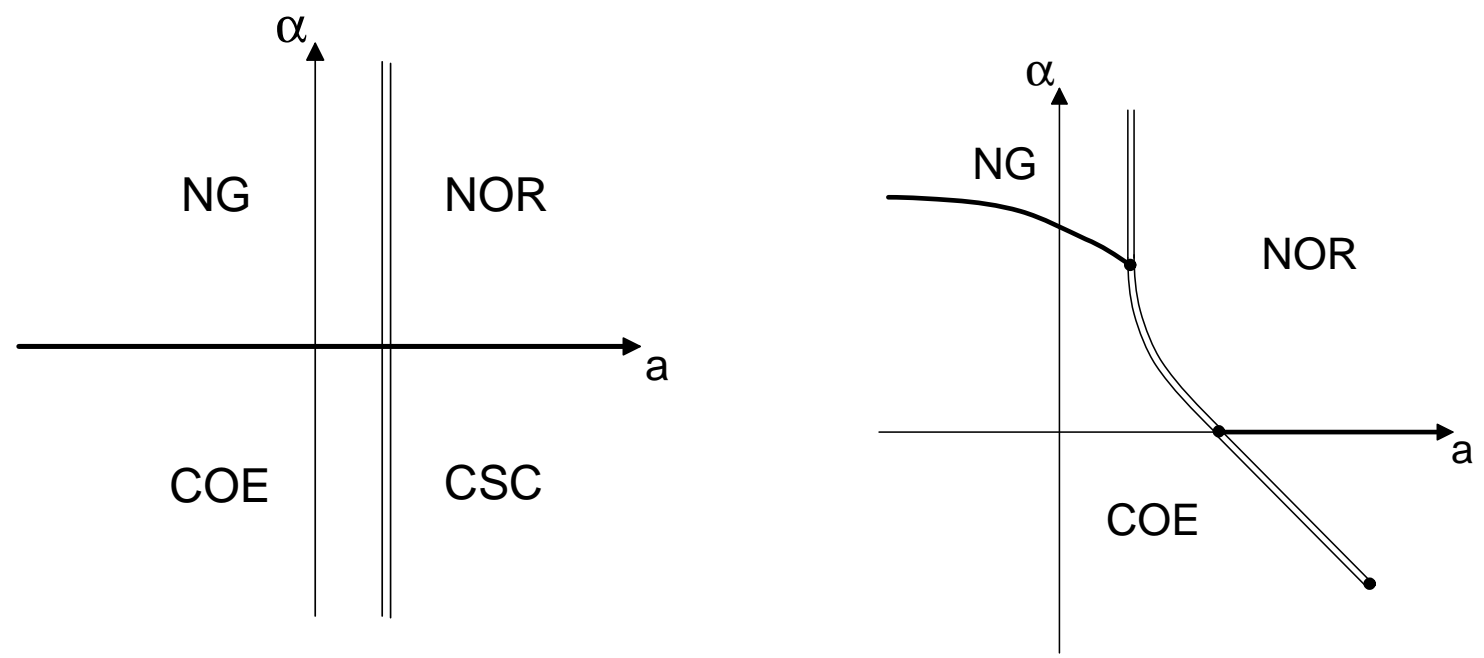

Figure 1. Phase structure in the $a-\alpha$ plane in the massless three-flavor case without $\gamma$-coupling (left) and with (right). Phase boundaries with a first order transition are denoted by a double line and second order by a single line.
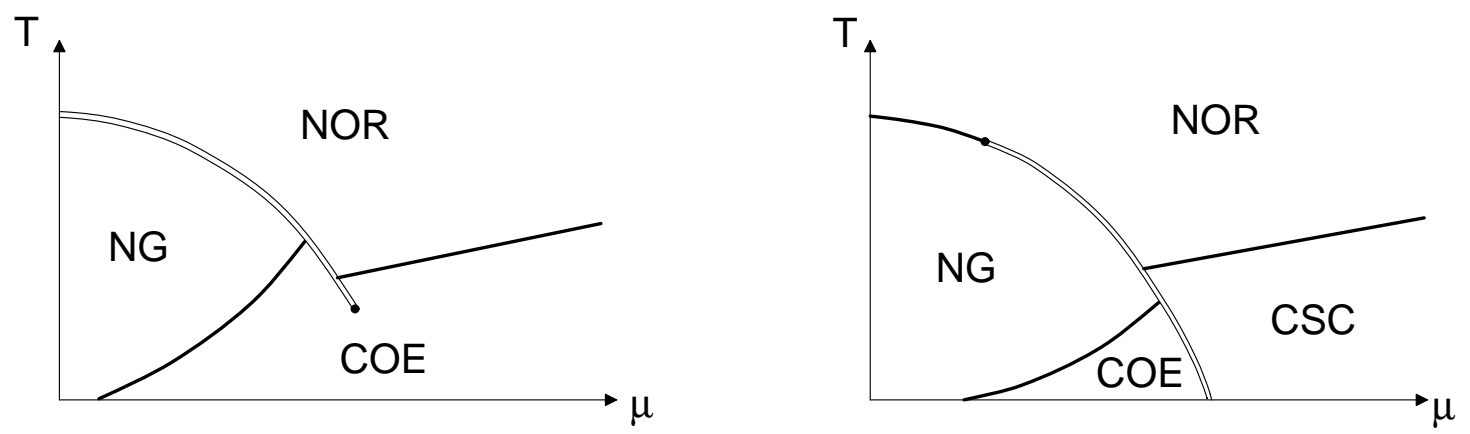

Figure 2. Schematic phase structure in the $T-\mu$ plane for the massless three-flavor case (left) and massless two-flavor case (right).

The phase structure with realistic quark masses (light up and down quarks and a medium-heavy strange quark) corresponds to a situation intermediate to the two cases in Fig. 3. There are two critical points in the diagram. The first one, near the vertical axis originally found by Asakawa and Yazaki [4], is driven by the quark mass which washes out the second order transition in high $T$ region. The one near the horizontal axis is our new critical point originating from the axial anomaly. The existence of the new critical point indicates that a smooth crossover takes place between the COE and CSC phases

$\S$ A similar critical point has been pointed out in [5] from the two-flavor NJL model. However, its origin is quite different from ours, since the axial anomaly does not produce the $\sigma d^{2}$ coupling in two-flavor case. 


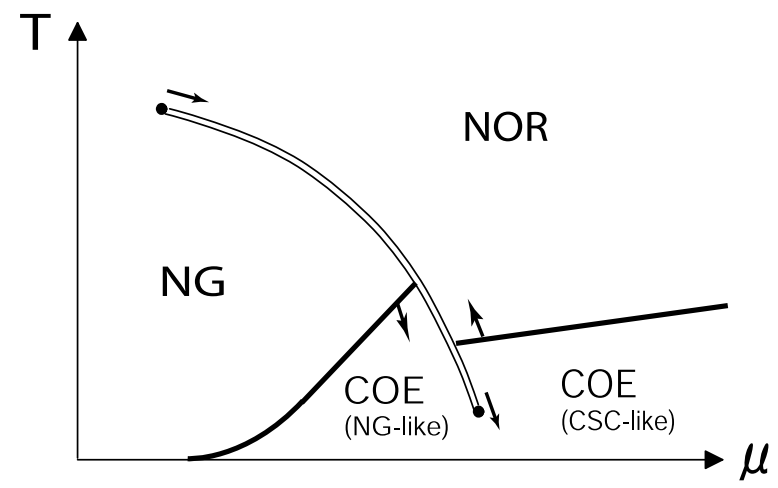

Figure 3. Schematic phase structure with realistic quark masses (light up and down quarks and a medium-heavy strange quark). The arrows show how the critical point and the phase boundaries move as the strange-quark mass increases toward the twoflavor limit.

\section{Conclusion}

We have utilized the model-independent GL approach to study the interplay between the chiral and diquark condensates. We found that a new critical point driven by the axial anomaly emerges and leads to a smooth crossover between the hadronic phase and the color superconducting phase at low temperature. Furthermore, in [2], we found that there is a smooth continuity of the elementary excitations, e.g., the ordinary pion $(\bar{q} q)$ in the NG phase and an exotic pion $(\bar{q} \bar{q} q q)$ in the CSC phase are mixed into a generalized pion satisfying a generalized Gell-Mann-Oakes-Renner relation in the intermediate density region. An interesting problem is to pin down the location of the new critical point from phenomenological models, and eventually from lattice QCD simulations.

\section{References}

[1] Hatsuda T, Tachibana M, Yamamoto N and Baym G 2006 Phys. Rev. Lett. 97122001

[2] Yamamoto N, Hatsuda T, Tachibana M and Baym G, paper in preparation.

[3] Schäfer T and Wilczek F 1999 Phys. Rev. Lett. 823956

[4] Asakawa M and Yazaki K 1989 Nucl. Phys. A 504 668; Barducci A et al 1989 Phys. Lett. B 231 463; Hatta Y and Ikeda T 2003 Phys. Rev. D 67014028

[5] Kitazawa M, Koide T, Kunihiro T and Nemoto Y 2002 Prog. Theor. Phys. 108929 